\title{
Simulation of railway track deterioration influenced by ballast stiffness and dry friction
}

\author{
J. Droździel \& B. Sowiński \\ Faculty of Transport Engineering, Warsaw University of Technology, \\ Poland
}

\begin{abstract}
It is known, that track deterioration (loss of its geometry) strongly depends on track support conditions, particularly on the ballast. In this paper a novel approach is presented to predict the evolution of track vertical irregularities due to vehicle-track dynamic interaction taking into account ballast stiffness with dry friction. An applied vehicle-track model is composed of a railway car and a track. The track is assumed to be a discrete system having a series of inertial elements, which are elastically coupled. The vertical ballast stiffness may fluctuate along the track and its damping is described by dry friction with the friction force depending on wheelset loading. In the track model, initial irregularities may be introduced to initiate the process of track deterioration and vertical track irregularities expansion. Simulation results are evaluated for a series of rail car passing and referred to other results.

Keywords: track, vehicle, dynamics, simulation, deterioration.
\end{abstract}

\section{Introduction}

Modern railway track is an important factor in providing a good transportation system. Large sums of money are dedicated to the railway track maintenance and renewal. Formerly, most attention has been given to the track superstructure (the rails, the fasteners and the sleepers), and less attention has been given to the substructure (the ballast, the subballast and the subgrade). Although these substructure components have a major influence on the track quality, less attention has been given to them because the properties of the substructure are more sophisticated then the properties of the superstructure.

This paper shall be restricted to examining the influence of the elastic and damping properties of the ballast on the track settlement and deterioration of its 
condition, in the form of vertical irregularities' changes. The presentation of a simulation model for track irregularities forecasting will be preceded by a short overview of the track's mechanical properties. For this reason, we've distinguished two subsystems (layers). The first one is a track grid composed of rails and sleepers attached to them, and the second is a layer of ballast together with the the subballast and the subgrade. The first subsystem serves for transmitting the load to the second one. Regarding the first subsystem, the knowledge of the track's geometry, type of the sleeper and vertical stiffness of the rails is sufficient for describing the model of the track settlement. Bearing in mind the considerable stiffness of the sleepers when it comes to bending, they may, in further considerations, be treated as perfectly rigid elements, pressed into the layer of ballast, as a result of loading by the passing train.

The analysis of the ballast operation, allows for describing the increase of the track's irregularities and its settlement [1]. As shown in the researches [1-3], in the process of the track's settlement, the most important factor is the ballast. The most vital function of the ballast is to maintain a stable position of the track by putting up resistance to the vertical and horizontal forces exerted on the sleepers, as well as reducing the loading caused by the sleepers onto the ground. The research indicates that deformations of the ballast layer contribute in a large extent to the track's settlement $[4,5]$. Froehling [6] in his paper on the causes of track's irregularities formation, points to the importance of the variable along the track of the vertical ballast's stiffness. There are assumed linear properties of the stiffness and damping for the ballast's layer.

It is also worth to mention the significant input of the experiment on the research done in the field of mechanical properties changes in the ballast, e.g. [6, 7].

The present work also acknowledges the crucial significance of the ballast in the process of settlement and track irregularities formation. Its mechanical model is represented in a one-layer structure with elastic and plastic properties, described by a discrete sequence of rheologic elements, comprised of a linear spring and a frictional damper The force of dry friction depends on the current vertical load of the track. It has been assumed that the vertical stiffness of the ballast along the track is constant.

\section{Model description}

As mentioned above, the track is treated as a superstructure, resting on the ballast. The track model is a mechanical system, where the deformation of ballast caused by loading results in the possibility of loosing the initial balance. Such properties can be noticed, for example, in systems with friction. Therefore, we assume that depending on the track loading process, the settlement and forming of the vertical geometrical irregularities of the track occur. Vertical irregularities of the track are reduced to its centre line.

In order to calculate the stiction force (no relative sliding), we do not introduce a continuous function as an equivalent (eg. arctan). To solve such a set, however, we propose an efficient algorithm. The problem is then to finding 
the equation of equilibrium (or equations for a system with many degrees of freedom), where the friction force shall have a value in a permissible area. The track model with a discrete support is shown below. It is a sequence of elementary models, elastically coupled (fig. 1). The equivalent mass of the elementary model depends on the sleeper's mass and on appropriate masses of rails, assigned to a particular sleeper. It may be used as a plane model, reduced to the track's centre line or as a model with two rails coupled through the sleepers.

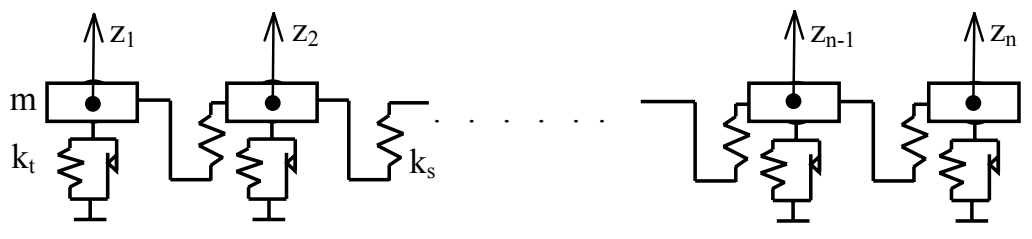

Figure 1: $\quad$ Track discrete model.

A rail vehicle is a passenger car with two bi-axle bogies (MD522). The car's model has 31 degrees of freedom (DOF); it means that every wheelset has 4 DOF (vertical and lateral motion, pitching and yawing), every bogie frame has 5 DOF and also 5 DOF for the body. Figure 2 shows a scheme of a car's model with marked DOF in vertical - longitudinal plane.

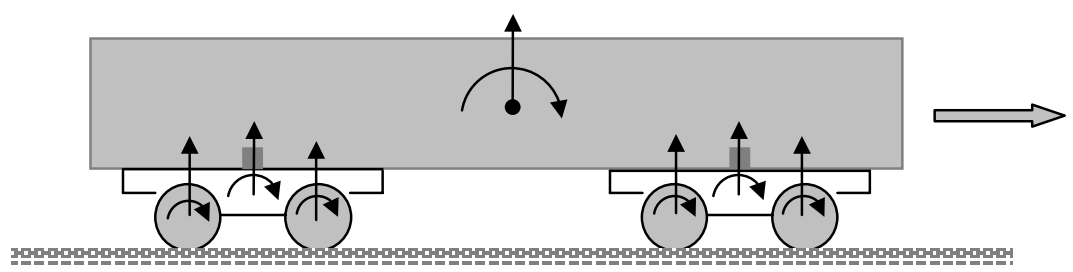

Figure 2: $\quad$ Rail vehicle model for vertical vibration.

A track's model shown in figure 1 was introduced for the purpose of describing the vertical interaction between the vehicle and the track. It has been assumed that the wheelset - track vertical force $Q_{i}(t)$ acting on $i$-th sleeper decreases linearly as the wheelset distance from the sleeper increases and reaches the zero value, when the wheelset is above the sleeper $(i+1)$. At the same time, this force increases in a linear way over the sleeper $(i+1)$ until it reaches $Q_{i+l}(t)$ value. The vertical force $Q_{i}(t)$ is determined by means of solving a system of differential equations for a vehicle - track interaction. For this purpose, a simulation program developed by the authors was used [8].

Here we concentrate on showing the system consisting of an equivalent concentrated mass of the sleeper and the rail, as well as massless, elastic and damping element with dry friction, representing the ballast. Such a system is influenced by a vertical force $Q(t)$ caused by moving vehicle. The equation of the track equivalent mass's vertical motion is represented below: 


$$
m \ddot{z}_{i}+k_{t} z_{i}+k_{s}\left(z_{i}-z_{i-1}\right)+k_{s}\left(z_{i}-z_{i+1}\right)+F_{i}=Q_{i}
$$

where

$\mathrm{i}=1,2, \ldots, \mathrm{n}$

$z_{i}-$ vertical coordinate of $i-t h$ track equivalent mass,

$m$ - track equivalent mass,

$k_{t}-$ ballast stiffness,

$k_{s}$ - coupling stiffness between repetitive track equivalent masses,

$F_{i}-$ dry friction force,

$Q_{i}$ - vertical axle load.

Figure 3 shows simplified characteristic of the friction force as function of the sliding velocity. The simplification consists in the assumption that the static friction force cannot exceed the kinetic friction force, as well as in accepting a constant kinetic friction force $F_{i}\left(\dot{z}_{i}\right)=$ const.

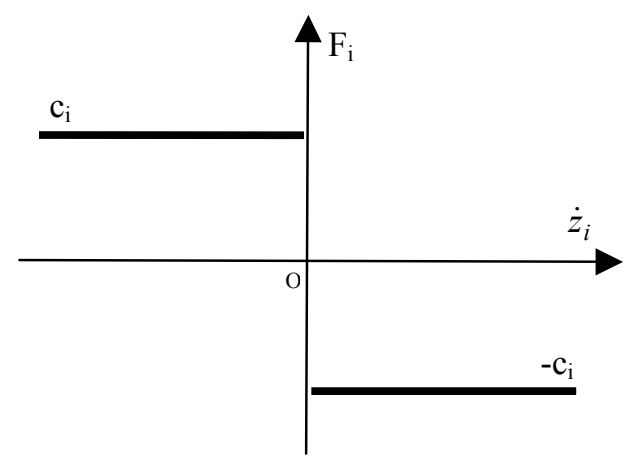

Figure 3: Friction force characteristics.

Mathematical description of this characteristic is presented below:

$$
F_{i}=\left\{\begin{array}{c}
-c_{i} \text { for } \dot{z}_{i}>0, \\
{\left[-c_{i},+c_{i}\right] \text { for } \dot{z}_{i}=0,} \\
+c_{i} \text { for } \dot{z}_{i}<0
\end{array}\right\}
$$

While the frictional damper is working, we may distinguish a phase of kinetic friction $\dot{z}_{i} \neq 0$ and a phase of static friction $\dot{z}_{i}=0$. In the set of equations (1), the kinetic friction force is clearly determined (see eq. (2)); however the static friction force $\dot{z}_{i}=0$ is a multiple valued and has to comply with the following condition: $\left|F_{i}\right| \leq c_{i}$.

In the static friction phase, two cases may occur:

1) velocity and acceleration meet the conditions: $\dot{z}_{i}=0 ; \quad \ddot{z}_{i}=0$

or

2) velocity and acceleration meet the conditions: $\dot{z}_{i}=0 ; \quad \ddot{z}_{i} \neq 0$,

According to equations (1), in the first case the static friction force is represented as follows: 


$$
F_{i}=Q_{i}-k_{t} z_{i}-k_{s}\left(2 z_{i}-z_{i-1}-z_{i+1}\right)
$$

In the second case, the force must be fulfill the equation of dynamic equilibrium, which means

$$
F_{i}=Q_{i}-m \ddot{z}_{i}-k_{t} z_{i}-k_{s}\left(2 z_{i}-z_{i-1}-z_{i+1}\right)
$$

In numerical calculations on floating force numbers, the realization of an assumption $\dot{z}_{i}=0$ does not give any chances for "success". Having this in mind, it may be assumed that $\dot{z}_{i}=0$, when $\left|\dot{z}_{i}\right|<\varepsilon$, where $\varepsilon$ is an assumed small number. Bearing in mind, that the simulation of the vehicle's motion on a $120 \mathrm{~m}$ long track shall require the introduction of 201 elements of a non-linear support for sleepers (every $0.6 \mathrm{~m}$ ). Finally, the effectiveness of the numerical methods of integration for a set of 402 first order differential equations has been tested. The tests show that the Runge-Kutta's method of the fourth order is a relatively fast one and brings the best results.

\section{Model testing and simulation results}

\subsection{Expansion of track vertical irregularities affected by a single welded joint of rails}

A single irregularity on the rails' weld was introduced as an element initiating the process of vertical irregularities expansion on a tested track. The irregularity (identical for both rails), is the vertical hollow of depth $0.5 \mathrm{~mm}$. Such a forced action has a transition character and results in an increased influence between the vehicle and the track. Successive passages on a tested track section may cause formation and expansion of vertical irregularities along the section of the track. In such a case, a process of growing amplitudes of those irregularities will start as well as the development of irregularity waves with different lengths. In simulation it was assumed that vertical stiffness and vertical damping in ballast are constant along the track and the damping is evoked by dry friction, described above. The characteristics of stiffness and damping for the vehicle's model are linear. In other authors' works $[6,9]$, the variable stiffness of the ballast was assumed, as well as a constant viscose damping along the road. Moreover, a logarithmic course of the track's settlement was accepted as function of the number of vehicle's passages. In the present work, the level of settlement and development of irregularities along the track is the result of a friction. The outcome of calculations was compared to measurements of track irregularities on a straight section of the PKP main line.

More important numerical data used in the simulation program are given below:

- length of the tested track section: 76,8m (includes 128 sleepers);

- number of wagon's passages over the tested section: $\mathrm{N}=700$;

- driving velocity: $30 \mathrm{~m} / \mathrm{s}$;

- vehicle's parameters like for a passengers wagon with MD 522 bogies;

- equivalent coefficient of the kinetic friction in ballast: 0.3;

- equivalent coefficient of the vertical stiffness of ballast: $102 \mathrm{MN} / \mathrm{m}$; 
- $\quad$ equivalent mass of a concrete sleeper together with UIC60 rails: $(280+120)$ $\mathrm{kg}$;

- vertical irregularity of rails on the welded rail joint: hollow type $-0.5 \mathrm{~mm}$, rail joint placed $60 \mathrm{~m}$ from the beginning of the track testing section.

Figure 4 shows the result of simulation in form of vertical irregularity of the track's central line and the result of measurement of his irregularity on the same section.

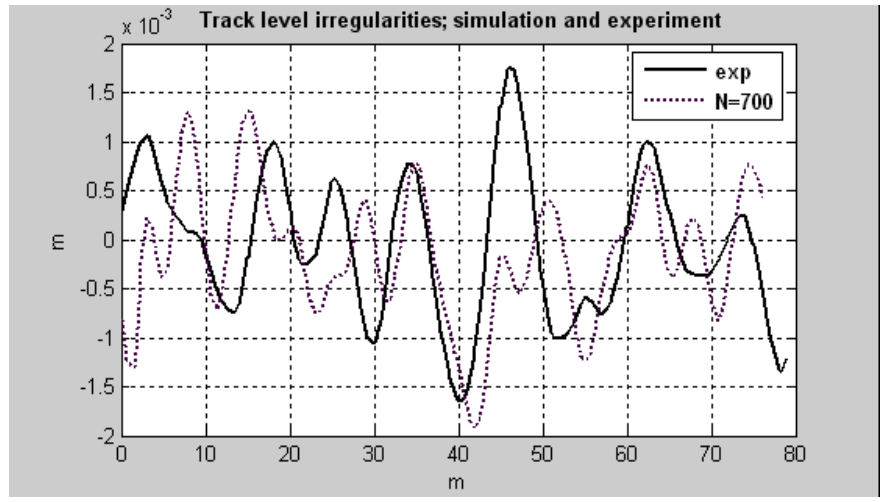

Figure 4: $\quad$ Track level irregularities; experiment and simulation.

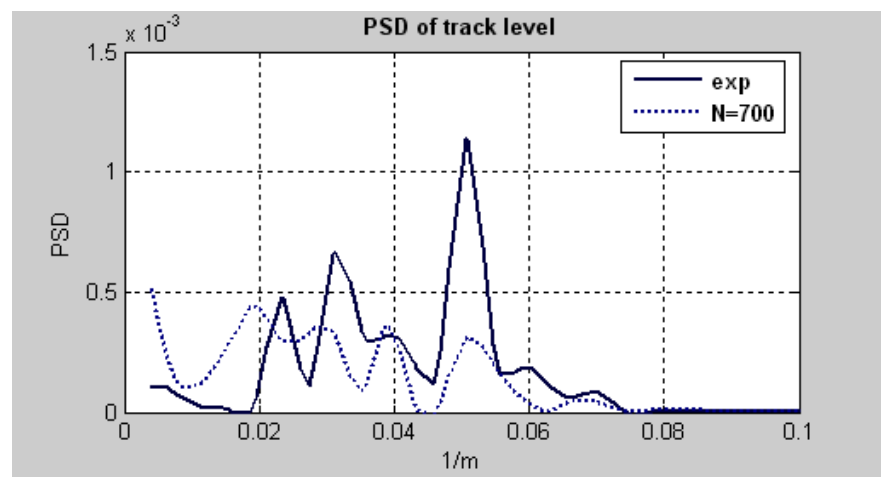

Figure 5: $\quad$ PSD of track level; experiment and simulation.

Figure 5 presents the comparison of the power spectral density (PSD) of these irregularities. The power spectral density of the vertical irregularities' signals show that the lengths of the dominant irregularities' waves are similar to each other. On the other hand, they differ by amplitudes. It must be mentioned however, that the measurements were taken on a track with a considerably greater accumulated track loading than 700 passages. The results of comparing the simulation and the experiment for several other track sections were also satisfying. 


\subsection{Track settlement simulation}

The simulation of the track settlement process is done by summing up the successive vertical displacements of the equivalent mass. The location of $i$-th equivalent mass corresponds to the location of $i$-th sleeper. After the passage of every car's wheelset, the vertical displacement of $i$-th track mass is calculated in comparison with the preceding location. The calculated displacement is added to (the sign of displacement may be either positive or negative) the value obtained after the passage of a previous wheelset.

The Figures $6 a \div 6 d$ show the results of the simulation of the track settlement process over one sleeper. The simulation was carried out for 40000 passages of wheelsets, which corresponds to 10000 passages of four-axle wagons. Two variants of static axle load on the track $(60 \mathrm{kN}$ and $100 \mathrm{kN})$ and two variants of ballast stiffness $(50 \mathrm{MN} / \mathrm{m}$ and $100 \mathrm{MN} / \mathrm{m})$ were considered. The properties of damping with dry friction were described by equivalent friction coefficients 0.2 and 0.3 . The parameters of four series of simulation are placed in table 1 .

\section{Table 1: $\quad$ Simulation parameters.}

\begin{tabular}{|l|l|l|l|}
\hline $\begin{array}{c}\text { Static axle } \\
\text { load }[\mathrm{kN}]\end{array}$ & $\begin{array}{c}\text { Ballast stiffness } \\
{[\mathrm{MN} / \mathrm{m}]}\end{array}$ & \multicolumn{1}{c|}{$\begin{array}{c}\text { Friction } \\
\text { coefficient }\end{array}$} & \multicolumn{1}{c|}{$\begin{array}{c}\text { Figure } \\
\text { No }\end{array}$} \\
\hline 60 & 50 & 0.2 & $6 \mathrm{a}$ \\
\hline 100 & 50 & 0.2 & $6 \mathrm{~b}$ \\
\hline 60 & 100 & 0.3 & $6 \mathrm{c}$ \\
\hline 100 & 100 & 0.3 & $6 \mathrm{~d}$ \\
\hline
\end{tabular}

Theoretical and experimental researches on the track settlement lead to determining several mathematical formulas. The most frequently used one is a logarithmic form given by Stewart [10]

$$
\varepsilon_{n}=a+b \log _{10} N
$$

where $\mathrm{a}$ and $\mathrm{b}$ are empirical constants. A modified formula was given by Froehling [6], basing on the experiment and a GEOTRACK software package. This formula has the form of

$$
\varepsilon_{N i}=\left[\left[K_{1}+K_{2}\left(\frac{k_{2 m i}}{K_{3}}\right)\right] \frac{P_{d y n}}{P_{r e f}}\right]^{w} \log _{10}(N)
$$

where

$K_{1}, K_{2}, K_{3}$ - are constants related to elastic properties of the track,

$k_{2 m i}$ - is an average track stiffness, measured on the i-th sleeper,

$P_{d y n}$ - is a dynamic loading on the wheel,

$P_{\text {ref }}$ - is a static loading on the wheel,

$w$ - empirically determined exponent,

$N$ - number of loading cycles (number of the wheelsets' passages). 

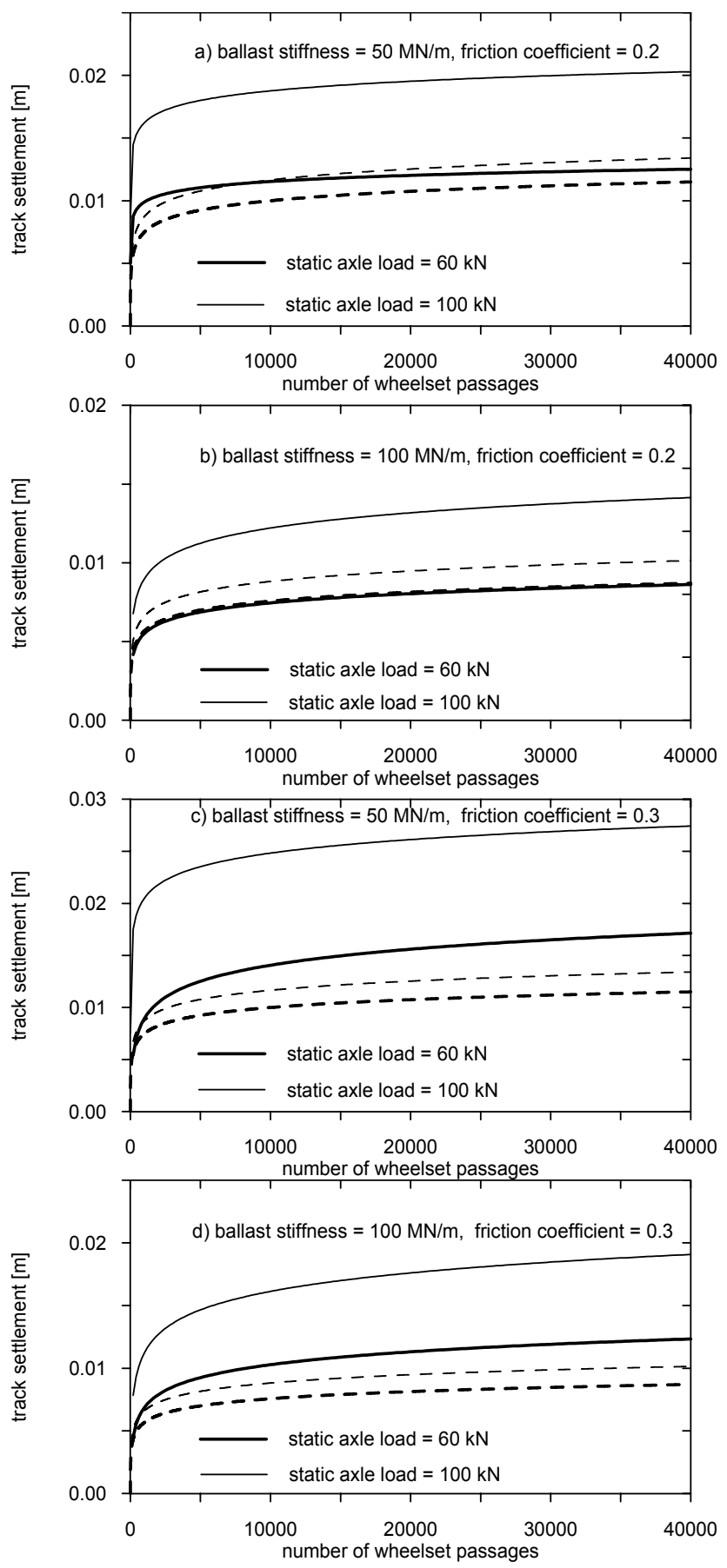

Figure 6: Track settlement on i-th sleeper. 
The curves of track settlement on i-th sleeper obtained from a simulation (ballast model with dry friction), are compared to curves determined in accordance with the equation (5). For the purpose of calculations, $k_{2 m i}$ equal $50 \mathrm{MN} / \mathrm{m}$ and $100 \mathrm{MN} / \mathrm{m}$ was assumed. However, it needs to be pointed out that the remaining parameters used for equation (6) were taken from work [6]. It has been done so, because the authors could not provide suitable parameters for the tested track section. Therefore, the comparison has, in this case, rather a qualitative meaning.

All the solid lines shown in Figure 6, illustrate the simulation results for the track settlement, considering dry friction in the ballast. Dashed lines, on the other hand, represent courses of the track settlement on the basis of formula (6). Thick lines (solid and dashed) correspond to the axle load of $60 \mathrm{kN}$, and thin lines (solid and dashed) - to the axle load of $100 \mathrm{kN}$.

Solid lines, representing the course of track settlement are obtained by approximations of sets of points obtained from the simulation. Formula (5) was the one used for approximation.

Certainly, the increase of the vehicle's axle load on the track causes the increase of this track's settlement. It happens in a greater extent for a greater friction coefficient. The increase of the ballast's stiffness changes considerably the level of settlement, using both the model with dry friction and the formula (6). The model with dry friction however, shows a stronger sensitivity when it comes to changes in axle load and changes of the ballast's stiffness. The higher friction coefficients cause the stronger track settlement.

\section{Conclusions}

The proposed model of the track settlement prediction, which concerns also dry friction, is an attempt of another description of this phenomenon, in relation to other works. It is a phenomenological model. It does not describe the ballast properties basing on soil mechanics equations. It is based on a relatively simple description of mechanical energy dissipation through dry friction and on conception of permanent deformations, such as settlement. In the further work, we are going to test a model with a non-linear stiffness of ballast and with a different friction description. The verification of the model shall be based upon a comparison of the track's settlement, obtained during the simulation and experiment. Introducing to the model variable ballast's stiffness along the track, it will be possible to predict the formation of track vertical irregularities.

\section{Acknowledgement}

The authors wish to acknowledge the financial support provided by the Ministry of Science and Higher Education (Project No 4 T12C 006 30).

\section{References}

[1] Esveld, K.C., Modern Railway Track, Second Edition. MRT-Productions, ISBN 90-800324, The Netherlands, 2001. 
[2] Wee Loon L., Mechanics of Railway Ballast Behaviour. PhD Thesis, The University of Nottingham, 2004,UK,

[3] Suiker A. S. J., The Mechanical Behaviour of Ballasted Railway Tracks. PhD Thesis, Delft University of Technology, The Netherlands, 2002.

[4] Sysak J. (red): Railway Roads. PWN, Warszawa, 1982 (in polish).

[5] Selig E.T. and Waters J.M., Track Geotechnology and Substructure management. Thomas Telford, London 1994.

[6] Froehling R. D., Low Frequency Dynamic Vehicle/Track Interaction: Modelling and Simulation. Vehicle System Dynamics, Suppl. 28, 1998, Swets \& Zeitlinger, pp. 30-46.

[7] Shenton M. J., Ballast Deformation and Track Deterioration. Track Technology Proc. Conf., London 1985, pp. 253-279.

[8] Droździel J., Sowiński B., et al. Dynamics of transient interaction between running gear of rail vehicle and track. Report of Research Project KBN, No 5 T12C 034 22, Warsaw University of Technology, 2004 (in polish).

[9] Froehling R. D., Scheffel, and Ebersoehn W., The Vertical Dynamic Response of a Rail Vehicle caused by Track Stiffness Variations along the Track. Vehicle System Dynamics Suppl. 25, 1996, Swets \& Zeitlinger, pp. 175-187.

[10] Stewart, H. E. Permanent strains from cyclic variable amplitude loadings. J. of. Geotechnical Engineering, ASCE, Vol. 112, No. 6, 1986, pp. 646660. 